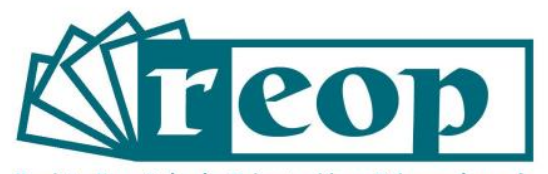

Revista Española de Orientación y Psicopedagogía

\title{
INCLUSIÓN ESCOLAR DEL ALUMNADO DE PRIMARIA Y SECUNDARIA CON ALTAS CAPACIDADES EN LA PROVINCIA DE SEVILLA (ESPAÑA)
}

\section{SCHOOL INCLUSION OF PRIMARY AND SECONDARY SCHOOL STUDENTS WITH HIGH ABILITIES IN THE PROVINCE OF SEVILLE (SPAIN)}

\author{
María José Navarro-Montaño \\ Universidad de Sevilla, Facultad de Ciencias de la Educación, Dpto. de Didáctica y Organización \\ Educativa. Sevilla, España \\ Elena Hernández de la Torre \\ Universidad de Sevilla, Facultad de Ciencias de la Educación, Dpto. de Didáctica y Organización \\ Educativa. Sevilla, España
}

\section{RESUMEN}

Este estudio tiene como objetivo conocer la atención educativa que se está ofreciendo actualmente al alumnado con Altas Capacidades Intelectuales en centros educativos públicos de educación primaria, secundaria y Asociaciones en Sevilla y provincia. Hemos indagado en la detección temprana y el abordaje de estrategias educativas, así como en la implicación familiar en el proceso educativo de estos estudiantes. La metodología seguida es de corte descriptivonarrativo a partir de un muestreo no probabilístico e intencional, combinando el enfoque cualitativo y el cuantitativo; se trata, por tanto, de una metodología mixta. Los instrumentos utilizados para la recogida de datos son entrevistas a equipos directivos de centros, orientadores, directores de Asociaciones y cuestionarios al profesorado. Los resultados destacan la falta de detección y apoyo en estadios iniciales por profesionales especialistas y la escasez de recursos en la trayectoria formativa de este alumnado; las conclusiones principales resaltan la necesidad de formación de profesionales y falta de asesoramiento a profesionales y a familias como principales implicados en su atención educativa. Las implicaciones para la práctica revelan que las Asociaciones colaboran con el profesorado en la elaboración de Programas de enriquecimiento, 
ofrecen información a las familias y atención individualizada en base a necesidades concretas, realizando tareas de orientación y sensibilización a nivel educativo, sanitario y universitario.

Palabras clave: orientación, educación inclusiva, altas capacidades intelectuales, asesoramiento, participación escolar.

\begin{abstract}
This study aims analyze the educational attention that is currently being offered to students with high intellectual abilities in primary and secondary public schools and Associations in Seville and province. We have inquired on early detection and the implementation of educational strategies to cater for their needs, as well as the involvement of the family in the educational process of these students. The research methodology is descriptive and narrative based on a non-probabilistic intentional sample, combining the qualitative and quantitative approach, therefore, it is a mixed methodology. The instruments used for data collection consist of interviews with school managing staff, counselors, and directors of Associations as well as questionnaires to school teachers. The results include lack of detection and support in early stages by specialized professionals and the lack of resources to teach these students. The main conclusions highlight the need for training of professionals involved in the identification and early assessment of students as well as the lack of advice to professionals and families as the main stakeholders involved in the educational care of these students. The implications for practice reveal that the Associations collaborate with teachers in the development of Enrichment Programs, offer information to families and individualized attention based on specific needs, carrying out guidance and awareness-raising tasks in educational, health and university contexts.
\end{abstract}

Key Words: educational guidance, inclusive education, high intellectual abilities, school participation.

\title{
Cómo citar este artículo:
}

Navarro-Montaño, M.J. y Hernández de la Torre, E. (2021). Inclusión escolar del alumnado de primaria y secundaria con altas capacidades en la provincia de Sevilla (España). Revista Española de Orientación y Psicopedagogía, 32(2), 150-169.

https://doi.org/10.5944/reop.vol.32.num.2.2021.31284

\section{Introducción}

El estudio de las Altas Capacidades (en adelante AACC), se sitúa en nuestra historia educativa más reciente en el comienzo del siglo XX vinculado al concepto de superdotado y al convencimiento de que estos estudiantes no necesitan atención educativa específica, ya que 
pueden aprender sin ayuda y superar sus propias dificultades de aprendizaje. La historia revela que la investigación sobre las AACC ha despertado gran interés en el panorama educativo internacional debido, entre otras razones, al creciente número de argumentos que apuntan hacia el impacto que tiene promover en el capital humano el desarrollo intelectual, cultural, social y económico de las naciones.

En nuestro contexto educativo, la LOGSE de 1990 contemplaba la sobredotación cognitiva vinculada a las necesidades educativas especiales; la LOCE de 2002 las consideraba como superdotación intelectual y en la LOE de 2006 aparece el término Altas Capacidades Intelectuales en sus artículos 76 y 77 . En el contexto educativo andaluz, el "Plan de actuación para la atención educativa al alumnado con necesidades específicas de apoyo educativo por presentar altas capacidades intelectuales en Andalucía" de 2011 recoge que, un estudiante presenta AACC cuando maneja y relaciona múltiples recursos cognitivos de tipo lógico, numérico, espacial, de memoria, verbal y creativo, o bien destaca especialmente o de manera excepcional, en el manejo de uno o varios de ellos. Las Instrucciones de 22 de junio de 2015, de la Dirección General de Participación y Equidad de la Junta de Andalucía, establecen el Protocolo de detección, identificación del alumnado con necesidades específicas de apoyo educativo y la organización de la respuesta educativa.

Un elevado número de alumnos/as con AACC pasan desapercibidos durante etapas educativas por desconocimiento en los centros o por falta de formación e información de los profesionales y familias. Ramos (2014, p.155) ha estudiado la naturaleza incluyente/excluyente del sistema educativo, desarrollando una perspectiva de análisis en la que considera que "es conveniente preguntarse cómo es posible que dicho sistema pueda excluir, si por su recorrido histórico contemporáneo, por norma constitucional, por derecho es incluyente..."; en este contexto Sáenz de Jubera y Chocarro (2019) estudian la atención a la diversidad desde la perspectiva del profesorado. En ocasiones las AACC no se detectan hasta que el estudiante comienza a manifestar comportamientos disruptivos, no ofreciéndole hasta entonces una respuesta educativa adecuada. Alrededor del $2 \%$ de la población posee AACC (15.876 niños/as de la población escolar en enseñanzas no universitarias, según Informe MEC, 2016), no obstante, la atención no parece ser adecuada y la falta de apoyo tiene implicaciones a nivel familiar, escolar y personal. Según las estadísticas, alrededor de un 35\% de este alumnado no alcanza el rendimiento académico esperado y casi la mitad no llega a la universidad, esto puede considerarse un fracaso del sistema educativo.

El potencial del alumnado con AACC no es consecuencia solo de su elevada inteligencia, también influyen una serie de variables personales y contextuales que pueden originar diferentes perfiles intelectuales (Hernández y Gutiérrez, 2014), y "factores sistémicos" (Velasco y QuirogaGarza, 2018) asociados a etapas concretas como es la adolescencia. Estos conceptos son utilizados con distintos criterios en nuestro contexto educativo, donde la ausencia de una definición oficial del término AC en la legislación educativa dificulta su estudio y el avance de la investigación. Es necesario unificar criterios respecto al concepto y diferentes perfiles intelectuales que engloba. Sastre-Riba (2014) se refiere a la necesidad de especificar una definición comprensiva de las AACC para minimizar estereotipos negativos y positivos (elitismo o privilegio). Plucker y Callahan (2014, p.176) recogen la teoría de Subotnik et al. (2012), entendiendo que "las AACC se pueden ver como de desarrollo ya que, en las fases iniciales, el potencial es la clave variable; en posteriores fases, la consecución es la medida; y en talentos más desarrollados, la eminencia es la base sobre la cual se consigue esta etiqueta". Esta teoría introduce el potencial como elemento clave, teniendo en cuenta el desarrollo del ser humano y enfatizando la contextualización de las AACC por englobar aspectos cognitivos y psicosociales.

El concepto AACC se considera un término paraguas ya que engloba otros conceptos, "nos referimos a superdotados, talentos, prodigios, genios, precoces, como expresiones diferenciadas de la alta capacidad como si de una paleta de colores se tratara, con matices y texturas" (Guirado, 2015, p.17). La inteligencia es personal y diversa, y se manifiesta de distintas formas en cada 
persona. El Consejo Superior de Expertos en Altas Capacidades concibe la superdotación como "resultado de la confluencia de la cognición (inteligencia e imaginación) con factores emocionales (intereses y motivación)" (Sánchez, 2013, p.75). Estas coincidencias, consideradas como orientación y guía para los educadores, son compartidas por alumnado que no alcanzan niveles de AACC, aunque si en un estudiante coincide un elevado número de ellas, las probabilidades de que el perfil sea AACC aumentan considerablemente. Hernández y Gutiérrez (2014, p.258) han investigado sobre el perfil afectivo, emocional y social del alumnado con AACC, desde dos perspectivas: inteligencia social-emocional y ajuste social y adaptación. Los autores entienden que estos estudiantes se perciben a sí mismos con una competencia social-emocional alta. El profesorado parece percibir al alumnado con AACC más adaptado, con más personalidad y mayores habilidades interpersonales, concluyendo que "no hay razones para pensar que la inadaptación y la alta capacidad intelectual sean variables relacionadas" (Hernández y Gutiérrez, 2014, p.258).

Con objeto de orientar la detección y evaluación de este alumnado, recogemos modelos centrados en su estudio. Los modelos explicativos siguen las aportaciones de Albes et al. (2013) y Torrego (2012), los modelos centrados en el estudio de las capacidades de Terman (1917) y Gardner (1985), considerados uno de los primeros intentos de definir las características del pensamiento excepcional son los llamados modelos psicométricos, cuya finalidad es medir las diferencias individuales relativas a las capacidades intelectuales (Sánchez, 2013), considerando la superdotación como una característica personal, de tipo innato. En su definición de la superdotación destacan el papel predominante de la inteligencia y del cociente intelectual, incluyendo posteriormente el de las aptitudes. Los modelos basados en el estudio del rendimiento de Renzulli (1978) y Gagné (1991) consideran las AACC como un potencial e incorporando otra serie de condiciones adicionales como pueden ser la creatividad o la motivación, de cuyo funcionamiento conjunto depende este alto rendimiento. Los modelos cognitivos de Sternberg (1985) se basan en las aportaciones de la psicología cognitiva, centrando su estudio en los procesos cognitivos, la elaboración y la gestión de la información. Por último, los Modelos de orientación sociocultural de Tannenbaum (1986, 1997) y Mönks (1992) que consideran que la cultura y la sociedad establecen lo que se considera talento especial, añadiendo además el contexto social y familiar como favorecedores del adecuado desarrollo del sujeto.

Entre las principales dificultades con las que se enfrenta la atención a las AACC está su detección que debería estar basada en criterios cualitativos más que cuantitativos. Sánchez (2013, p.75) señala que en la detección y diagnóstico "deben participar profesionales con competencias sanitarias, no solo educativas". El criterio fundamental en el que se ha basado la detección y diagnóstico de las AACC ha sido tener una inteligencia superior a la media, aunque autores como Hernández y Gutiérrez (2014) señalan que, durante las últimas décadas, este estudio se ha caracterizado por una incorporación de distintas variables cognitivas más allá de la inteligencia. Algunas de las características han sido estudiadas por diversos investigadores, (Albes et al. 2013; Torrego, 2012) que entienden que entre los estudiantes con AACC podemos encontrar una gran diversidad con características comunes, con lo que no constituye un grupo homogéneo y su atención tiene que ser diferenciada y estar basada en necesidades educativas concretas, motivación e intereses. Al respecto, Núñez et al. (2014) han analizado las diferencias en las estrategias y patrones motivacionales manifestados por estos estudiantes en función del significado otorgado a la atención a la diversidad.

La importancia de la evaluación psicopedagógica en este contexto y del asesoramiento debe ir acompañada de un diagnóstico cuyo enfoque sea compresivo y holístico que permita conocer las necesidades específicas de este alumnado para establecer la respuesta educativa más adecuada (Kim, 2014). Sánchez (2013), entiende la evaluación psicopedagógica con la finalidad de diagnóstico de las AACC, en la que además de las pruebas psicométricas, es necesaria la información de familias y profesorado. Asimismo, Hernández y Gutiérrez (2014) afirman que se están desarrollando métodos y procedimientos de evaluación complementarios a las pruebas de inteligencia y aptitudes intelectuales que permiten realizar un análisis más completo de la 
naturaleza multidimensional del constructo. Entre las iniciativas más novedosas en materia de identificación, está la adaptación del Talent Search Model al contexto educativo español (Touron y Touron, 2011), ya que puede seleccionar a candidatos con talento verbal o matemático y su mayor ventaja es que permite superar el efecto techo que caracteriza a otras pruebas de inteligencia y aptitud intelectual.

La intervención educativa debe proporcionar experiencias, vivencias y recursos para que los estudiantes puedan desarrollar y expresar su potencial en todas las dimensiones (Albes et al., 2013; Palomares y García, 2016). También Vallejo y Morata (2015) afirman que el estado intelectual es producto de la interacción entre los factores innatos y el entorno que envuelve al sujeto, por lo que favorecer el desarrollo personal pleno es una de las funciones principales del docente que, además, debe facilitar un ambiente de aprendizaje que promueva el desarrollo de las capacidades. Para Martínez y Guirado (2014, p.34), es importante "favorecer la toma de conciencia de las necesidades educativas específicas del alumnado con altas capacidades intelectuales y del papel crucial de la escuela y la familia en el desarrollo de dichas capacidades", para orientar los programas educativos en base a necesidades e intereses. En la actualidad, contamos con algunas claves para orientar la respuesta educativa a través de diferentes procedimientos como son los programas de enriquecimiento educativo, el agrupamiento flexible 0 la aceleración escolar, aunque, son escasos los estudios que aportan resultados empíricos sobre la eficacia de estas actuaciones.

Algunos estudios, programas y cursos recientes aportan datos específicos respecto a la atención a estudiantes con AACC con la finalidad de mejorar su práctica educativa. Entre estos se encuentran los siguientes: estudio sobre altas capacidades creativas y bienestar psicológico (Kroesbergen et al., 2016) en Holanda, estudio sobre aspectos socio-emocionales y programas de aceleración (Gronostaj et al., 2016) en Alemania, estudio sobre recursos socio-emocionales y funcionales (Salmela y Määttä, 2015) en Finlandia, estudio sobre orientación educativa adecuada (Mudrak y Zabrodska, 2015) en República Checa, estudio sobre la Teoría Rem (Seaton et al., 2015), programas de enriquecimiento (Kim, 2016), programa educativo para estudiantes talentosos (Rasmussen y Rasmussen, 2015), cursos de aceleración (McClarty, 2015) y desempeño de los estudiantes más capaces para la matemática en pruebas de rendimiento concretas como es el caso de BECOMA (García, 2016), entre otros.

La presencia en los centros de alumnado con AACC, la necesidad de detección temprana, el empleo de estrategias de atención educativa en los centros escolares y programas adecuados, son los temas de interés para el estudio que presentamos, por lo que entendemos justificada su pertinencia, adecuación y actualidad. Recientemente, Azorín (2018) partiendo del concepto de equidad, hace alusión a un sistema educativo y social basado en criterios igualitarios para todos los estudiantes; la autora reflexiona sobre las interacciones que se producen en las escuelas entre las escuelas y más allá de las escuelas (Ainscow et al., 2013). En este marco de acción común, las escuelas han ser organizaciones que reflexionen sobre sus propias prácticas desde una perspectiva ecológica en la que el estudio del centro en su contexto más inmediato adquiere especial relevancia. La atención educativa y social a los estudiantes con AACC se contextualiza en el marco de la equidad, es decir se trata de ofrecer a cada estudiante la atención que necesita en base a sus necesidades educativas, en un contexto educativo inclusivo. En este sentido, investigaciones recientes ponen de manifiesto la necesidad de eliminar las barreras de la educación inclusiva, realizando propuestas sobre facilitadores para su desarrollo, como afirman González-Gil et al. (2019) que realizan este análisis partiendo de las percepciones del profesorado sobre la educación inclusiva. En esta misma línea de trabajo, Orozco y Moriña (2019) investigan sobre las prácticas docentes cuyo propósito es conseguir una pedagogía más inclusiva partiendo del análisis de las voces del profesorado. También Ferrer (2019) se refiere a la necesidad de implementar buenas políticas y prácticas para la educación inclusiva.

En relación con la intervención orientadora, investigaciones como la de Sánchez-Escamez (2017) estudian la identificación de las AACC desde el Departamento de Orientación Educativa 
con la implicación del profesorado y Morales (2017) también investiga sobre el reto que supone para los profesionales de la orientación educativa, la intervención orientadora a las AACC, Asimismo, Garcerán (2019) estudia la importancia de la orientación a las familias de estudiantes con AACC y Fernández-Batanero (2020) se ha referido a la orientación educativa vinculándola a los aprendizajes transversales. Por tanto, las prácticas educativas en base a criterios equitativos han de ser las bases de la orientación de los estudiantes con AACC hacia modelos educativos inclusivos desde los que responder a sus necesidades. Todo ello tiene importantes implicaciones para el desarrollo de la intervención orientadora de estos estudiantes en los centros educativos.

\section{Método}

La metodología que se ha utilizado es descriptiva-exploratoria. En este estudio no se formulan hipótesis, ya que de acuerdo con Hernández-Sampieri et al. (2014, p.104) en "los estudios de alcance exploratorio, no se formulan hipótesis". El carácter de este diseño nos ha llevado a optar por el desarrollo de este enfoque metodológico. Como señalan Hernández-Sampieri, et al. (2014, p.534), estos métodos "implican la recolección y el análisis de datos cuantitativos y cualitativos, así como su integración y discusión conjunta para realizar las inferencias producto de toda la información recabada (metainferencias) y lograr un mayor entendimiento del fenómeno bajo estudio".

El objetivo principal del estudio es conocer y analizar en profundidad la situación educativa respecto a la atención escolar al alumnado con AACC en centros educativos públicos de primaria y secundaria, así como Asociaciones en la provincia de Sevilla. Basándonos en este objetivo principal, planteamos los siguientes:

- Detectar y contactar con profesionales de una muestra de centros educativos públicos, concertados y privados, así como Asociaciones que trabajan con alumnado con AACC de primaria y secundaria.

- Analizar las opiniones y necesidades formativas del profesorado en relación con la atención educativa que se ofrece al alumnado con AACC.

- Identificar las aportaciones y evidencias respecto a la detección, atención y respuesta escolar que ofrecen los centros educativos y Asociaciones que atienden a este alumnado.

\section{Muestra}

Este estudio tiene una orientación descriptiva y se ha desarrollado a partir de entrevistas a 4 equipos directivos, 2 directores de asociaciones y 2 orientadores de centros, así como cuestionarios a 23 tutores de primaria y secundaria. Para la selección y descripción de la muestra, caracterizada por su disposición a colaborar, se ha empleado un muestreo no probabilístico de tipo intencional, ya que al ser un estudio donde se ha priorizado la metodología cualitativa, "el investigador selecciona a los individuos en función de que puedan aportar la información necesaria para comprender comportamientos en un determinado contexto" (Clares, 2016, p.191). La muestra es intencional en base a los criterios de disponibilidad de los sujetos participantes, su implicación en el trabajo con este alumnado y la participación voluntaria en el mismo. Los 
principales argumentos que justifican la elección de este diseño metodológico son: por un lado, que el estudio de las AACC es un tema que debería vincular la investigación con la práctica educativa, y por otro, que uno de los propósitos del estudio es describir cuál es la situación de la atención y respuesta a este alumnado en los centros.

El cuestionario se ha enviado a 23 profesores/as de primaria y secundaria relacionados con la atención a este alumnado. Los participantes que constituyen la población de la entrevista han sido equipos directivos de 2 Centros de Secundaria, 2 de Primaria, y 2 directores de Asociaciones. Los códigos de análisis utilizados son los que presentamos a continuación:

- IES-LEB: código IES1.

- IES-LLA: código IES2.

- CEIP-SIS: código CEIP1.

- CEIP-CAR: código CEIP2.

- ASO-CAD: código ASO1.

- ASO-ADO: código ASO.

\section{Instrumentos}

El proceso de validación del cuestionario responde a la siguiente secuencia:

1ํ Elaboración de una tabla de doble entrada que recoge, por una parte, el cuestionario sobre AACC cuyos ítems se presentan organizados en base a las cuatro grandes dimensiones con objeto de situar al evaluador/a y por otra, se solicita la titulación, especialidad y años de experiencia.

2ํㅡㄹ El evaluador/a tiene que valorar la pertinencia/relevancia del ítem y la adecuación/claridad del ítem de 1 a 5 , siendo 1 nada de acuerdo y 5 totalmente de acuerdo, además se solicitan sugerencias para mejorar el instrumento.

3ํㅡㄴ Envío del instrumento por correo a la muestra seleccionada para validarlo. Han participado 4 profesores/as (2 de Primaria y 2 de Secundaria) y 2 orientadores/as. Los criterios para seleccionar la muestra fueron principalmente que los perfiles profesionales de los seleccionados fueran afines a la investigación, así como la disponibilidad para participar.

4ํㅗㄹ A partir de la recepción de las tablas para validar el instrumento hemos procedido a realizar las modificaciones sugeridas por los evaluadores/as (aspectos formales y sustitución de algún término para clarificar y mejorar la comprensión del ítem) y elaborado el instrumento final.

Las 12 preguntas del cuestionario se han graduado de 1 al 5 en sus respuestas, siendo el 1 equivalente a "nada de acuerdo", y el 5 "totalmente de acuerdo"; incluyendo una última pregunta de respuesta abierta. El instrumento se compone de 12 ítems y 4 dimensiones (Figura 1):

- Dimensión 1-Información/formación AACC (ítems 4-5).

- Dimensión 2-Atención educativa/respuesta a las necesidades (ítems 1-2-3-6-9).

- Dimensión 3-Socialización /relaciones sociales (ítems 10-11-12).

- Dimensión 4-Recursos humanos (ítems 7-8). 
Las preguntas que constituyen la entrevista realizada a Equipos Directivos/orientadores en centros y Asociaciones son las siguientes:

- 1. ¿Cree que en su centro se atiende a la diversidad del alumnado?

- 2. ¿Cuáles son las situaciones de diversidad que se dan con mayor frecuencia?

- 3. Con relación a ello, ¿se responde a las necesidades educativas del alumnado con altas capacidades?

- 4. ¿Qué profesionales han intervenido en el informe de diagnóstico?

- 5. ¿Qué estrategias y recursos educativos se utilizan para atender a estos alumnos?

- 6. ¿Existen Programas o adaptaciones curriculares para ellos?

- 7. ¿Qué profesorado los atiende?

- 8. ¿De quién son responsabilidad este alumnado?

\section{Procedimiento}

Las fases en las que se ha desarrollado este estudio se concretan en las siguientes:

- Fase 1: Preliminar: detección y contacto inicial con centros públicos, concertados y Asociaciones que atienden al alumnado con AACC, revisión de literatura específica sobre atención y respuesta educativa a este alumnado.

- Fase 2: Entrevistas con equipos directivos de centros y asociaciones: entrevista en centros y Asociaciones con objeto de obtener una visión general de las actuaciones y respuestas educativas de los centros a este alumnado y cuestionario a profesorado de centros escolares de primaria.

- Fase 3: Elaboración de resultados y conclusiones respecto a la atención a las AACC: análisis exhaustivo de las aportaciones mediante los instrumentos de recogida de datos en este estudio.

\section{Análisis de datos}

El análisis de los datos de entrevistas se ha llevado a cabo a través del análisis de contenido de Bardín (1996), generando a partir de este análisis las categorías y códigos ya clásicos en este tipo de investigaciones; el cuestionario se ha analizado con el Programa SPSS.23 de análisis de datos aplicando como técnica la puntuación media.

El proceso de análisis responde a la siguiente secuencia: en primer lugar, se han explorado los datos del cuestionario para conocer el estado de la cuestión respecto a la atención que recibe este alumnado; a continuación, se ha procedido a realizar entrevistas con una doble finalidad: por una parte, profundizar sobre aspectos de interés para el estudio tras la aplicación del cuestionario y por otra parte, obtener datos concretos sobre la atención a las AACC en centros y Asociaciones. 
En relación con la elección del enfoque mixto en el análisis de datos, se justifica porque pretende indagar y definir de forma concreta y contextual las AACC. El propósito del cuestionario es valorar el conocimiento del profesorado respecto a las AACC, así como los facilitadores y obstaculizadores que dificultan su respuesta educativa. El análisis de los datos recogidos de la entrevista nos ha permitido seleccionar aquellas dimensiones más relevantes para su estudio en mayor profundidad. Por otra parte, la orientación descriptiva-narrativa que caracteriza el análisis de datos se justifica porque "la investigación cualitativa se enfoca en comprender los fenómenos, explorándolos desde la perspectiva de los participantes en un ambiente natural y en relación con su contexto" (Hernández-Sampieri et al., 2014, p.358); y porque el tema de estudio ha sido débilmente explorado. A partir de la entrevista abierta se ha realizado un profundo análisis de los contenidos de las respuestas de los Equipos Directivos a las cuestiones planteadas.

\section{Resultados}

El análisis de datos obtenidos mediante el cuestionario y las entrevistas muestran los resultados que a continuación se detallan.

\section{Análisis de los datos del cuestionario por ítems}

En relación con el cuestionario presentamos la siguiente Figura:

\section{Figura 1}

Ítems y respuestas del profesorado al Cuestionario

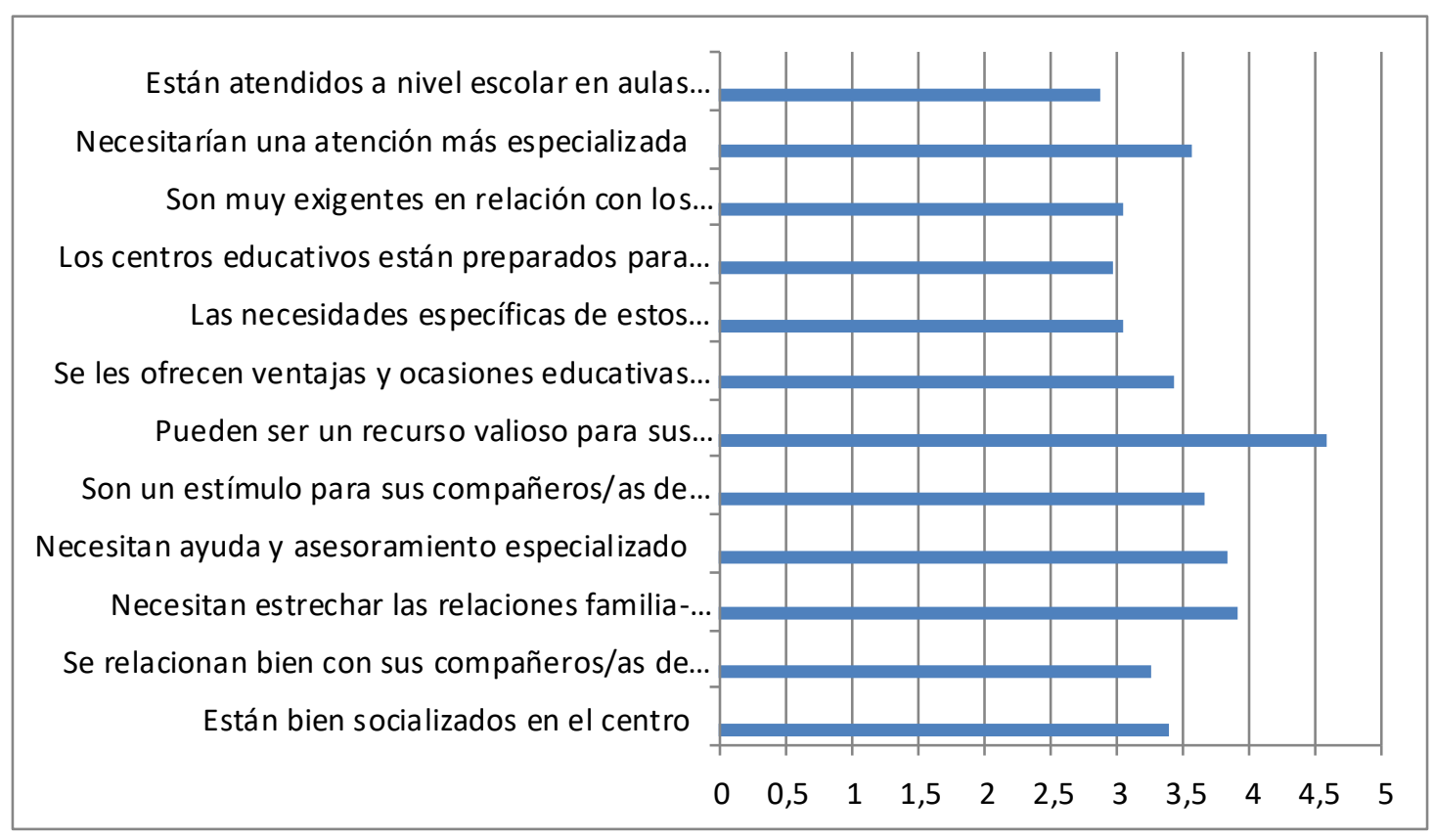

Fuente: Elaboración propia 
El análisis de los datos en función de las respuestas a los ítems del cuestionario es el siguiente:

Ítem 1: Están atendidos a nivel escolar en aulas ordinarias para apoyar de manera adecuada a estos estudiantes: la puntuación media es de 2,87 , lo que indica un número no demasiado alto de respuestas afirmativas, con tendencia a la baja.

Ítem 2: Necesitarían una atención más especializada: la puntuación media a esta pregunta es de 3,57, por lo que la mayoría de los participantes cree que la atención al alumnado con AACC debería ser más especializada.

Ítem 3: Son muy exigentes en relación con los contenidos y ritmos de aprendizaje: en esta pregunta, los resultados se mantienen en un neutral 3,04, lo que vendría a indicar que no todos los participantes creen que este alumnado sea especialmente exigente.

Ítem 4: Los centros educativos están preparados para ofrecer una atención educativa adecuada a sus necesidades: la respuesta media de 2,96 queda por debajo de la media, considerando el profesorado que no todos los centros están preparados para atender adecuadamente las necesidades del alumnado con AACC.

Ítem 5: Las necesidades específicas de estos estudiantes son ignoradas por algunos centros educativos: a pesar de que la puntuación media es de 3,04, ligeramente por encima de la media, indica que algunos centros educativos ignoran las necesidades específicas del alumnado con AACC.

Ítem 6: Se les ofrecen ventajas y ocasiones educativas adecuadas a su nivel: las respuestas afirmativas son superiores a la media, de un 3,43.

Ítem 7: Pueden ser un recurso valioso para sus compañeros de clase: muestra un mayor grado de unanimidad entre los entrevistados. La puntuación final es de 4,57, casi la máxima, lo que indica la valoración positiva que hace el profesorado del papel del alumnado con AACC en relación con el resto de los compañeros.

Ítem 8: Son un estímulo para sus compañeros/as de clase: existe mayor acuerdo entre el profesorado, obteniendo una puntuación media de 3,65.

Ítem 9: Necesitan ayuda y asesoramiento especializado: la puntuación media de 3,83 apunta a la necesidad de una ayuda y asesoramiento especializado para atender al alumnado con AACC.

Ítem 10: Necesitan estrechar las relaciones familia-escuela: una puntuación media de 3,91 apoya la necesidad de coordinación entre las familias y las escuelas para atender al alumnado con AACC.

Ítem 11: Se relacionan bien con sus compañeros/as de clase: a pesar de haber reconocido la importancia del estímulo que el alumnado con AACC supone para los compañeros, al valorar las relaciones ésta es menor, quedando en un 3,26.

Ítem 12: Están bien socializados en el centro: un 3,39 es la nota media de la socialización del alumnado con AACC en sus centros educativos, ligeramente por encima de la media. 


\section{Análisis de datos del cuestionario por dimensiones}

Presentamos el análisis de las cuatro dimensiones en las que hemos agrupado los ítems:

Dimensión 1-Información/formación AACC (ítems 5-4): destacamos que los centros educativos se encuentran en el proceso de adecuar su atención al alumnado con AACC en lo que se refiere a la formación del profesorado, al diagnóstico y a los recursos y estrategias con los que se les atiende.

Dimensión 2-Atención educativa/respuesta necesidades (ítems 1-2-3-6-9): en la actualidad no existe una atención adecuada a este alumnado, necesita una mayor especialización; sin embargo, sí obtienen ventajas, aunque con ayuda y asesoramiento especializado.

Dimensión 3-Socialización/relaciones sociales (ítems 10-11-12): el profesorado opina que es necesario mejorar la coordinación de la relación entre familia-escuela, y estimular la relación con los compañeros de clase, aunque está bien valorada y progresan en su socialización.

Dimensión 4-Recursos humanos (ítems 7-8): el profesorado reconoce que este alumnado es un recurso valioso en clase (un ejemplo es la elaboración de actividades concretas: el "periódico de clase") como estímulo para sus compañeros.

\section{Análisis de entrevistas}

Las entrevistas han sido analizadas en profundidad mediante los siguientes códigos:

- $\quad$ Cod1. ADI: Atención a la diversidad /AACC en los centros.

- $\quad$ Cod2. DRE: Detección y respuesta escolar a las AACC.

- $\quad$ Cod3. PIO: Profesionales implicados: profesorado y orientadores.

- $\quad$ Cod4. EMR: Estrategias, medidas y recursos educativos.

- $\quad$ Cod5. PFA: Papel de la familia.

- $\quad$ Cod6. OBB: Obstáculos y barreras.

Presentamos los resultados del análisis de las entrevistas en base a los códigos:

Cod1. ADI: Los equipos directivos reconocen la diversidad en los centros educativos, aunque se trabaja más con alumnado de apoyo escolar que de AACC, "no trabajamos con los que destacan, siempre se trabaja desde los que necesitan apoyo" (IES1). Admiten que se atiende a todo el alumnado, es "atención individualizada a cada alumno de acuerdo con sus necesidades" (IES1), pero en realidad "lo que se hacen son adaptaciones curriculares por encima y eso implica actividades escolares propiamente dichas" (CEIP1). Los Departamentos detectan las AACC en determinadas materias, "en el Departamento intentamos eso, detectamos alumnos en matemáticas" (IES2). Reconocen que las AACC existen diciendo "sí que tenemos, no tanto como de necesidades educativas especiales, hay bastantes" (IES1).

Aunque la normativa indica que la atención al alumnado con AACC debe ser en los propios centros, los Equipos Directivos afirman que atienden al alumnado con necesidades educativas especiales por la mañana y como refuerzo para los de AACC por la tarde, "tenemos refuerzo y ese mismo refuerzo para los de AACC por la tarde, vienen los que tienen este retraso y los que van muy avanzados" (IES1). Indican que hay más alumnado que necesita refuerzo educativo, además, 
al profesorado le cuesta atender a los estudiantes con AACC, "el profesorado dentro de la clase lo trabaja muy poco, le cuesta mucho trabajar con AACC y le cuesta mucho trabajar con el niño que necesita refuerzo educativo también" (IES1).

Algunos centros deciden, junto con la tutora, que "se le proponga algún trabajo que quieran.... no hay una programación dirigida para ellos, para las inquietudes que tienen" (CEIP1). La atención se realiza "a demanda", "dependiendo del niño se le da un tratamiento u otro, la mayoría son actividades complementarias" (CEIP2), "con estas edades si tienen un interés que les motive, que sea ahí donde trabaje..." (CEIP1). Existe sin embargo conciencia de atención al alumnado con AACC, "nosotros trabajamos la atención a la diversidad y las AACC (...) con adaptación curricular, programación y trabajamos por la tarde de forma particular para ayudar a estos niños" (IES1).

Cod2. DRE: la detección es un problema de los centros, recayendo en el profesorado de aula, aunque.... "es quién lo detecta en clase.... sus actitudes son diferentes, la forma de pensar, presentar los problemas, las preguntas que hacen" (IES2). En otras ocasiones son los orientadores de los Equipos de Orientación Educativa los que confirman unas AACC detectadas previamente por el profesorado. La detección se incluye dentro de la evaluación psicopedagógica, "se hace en el centro.... son niños diagnosticados o que los tutores aprecian cierta habilidad, en cálculo, en expresión lingüística, en creatividad" (IES1). En general es el profesorado quien lo detecta, "detectarla en clase y a través del tutor o el profesor que lo deriva al equipo, al departamento de atención a la diversidad, y a partir de ahí se trabaja y se siguen los procedimientos establecidos en el protocolo" (CEIP2).

Los profesores/orientadores se enfrentan para su detección al tipo de capacidad que presenta el alumnado, "tienes que hacer muchas evaluaciones para ver si es talento complejo, talento simple" (IES1). Cuando se realiza esta detección cambia la situación, "hemos trabajado bien con ellos al nivel que necesitaban, algunos han necesitado adelantar un curso, otros han necesitado un refuerzo en su creatividad..." (IES1).

Respecto a la respuesta educativa y escolar a las AACC, la Consejería de Educación "da una serie de opciones, hacer una adaptación curricular o flexibilizar el horario, pasar de nivel o pasar un curso. Hay unas medidas que son las que aplicamos los centros" (IES1). A nivel institucional, "te proponen una forma de detectar esas necesidades, clasificar a los niños, censarlos, unos vienen censados del cole, otros los evaluamos aquí, tú tienes que marcar en Séneca" (IES1). Las Asociaciones están cubriendo la detección y respuesta a las necesidades educativas, resuelven sus necesidades de formación a demanda, "la finalidad es que los niños tengan un lugar donde realizar actividades y fomentar sus intereses y sus capacidades, y también intentar que haya leyes de prevención e identificación" (ASO1). La labor de las Asociaciones tiene importancia como formación y detección ".... no saben la diferencia entre sobredotación y talento y la miden mediante $\mathrm{Cl}$ cuando la Federación Andaluza no diferencia por puntuación de $\mathrm{Cl}$ sino por otro tipo de valoración" (ASO1); no manejan el concepto de "síndrome de disincronía" en el diagnóstico, "sí vemos que se adelantan a la adolescencia cognitivamente pero emocionalmente no están preparados para crecer tan rápido, esa disincronía hay que trabajarla bien" (ASO1). En las Asociaciones se ofrece una respuesta debido a que en los centros ordinarios no se dan alternativas, "una vez evaluados no se les daba nada y empezamos a investigar qué es lo que se hacía en otros centros a nivel de Programas de enriquecimiento" (ASO2).

Cod3. PIO: los profesionales que atienden a este alumnado son profesorado y orientadores, diferenciando entre atención de los centros, equipos de orientación y Asociaciones. El tutor es el máximo responsable, explora su funcionamiento en el aula, currículum y comportamiento en clase/centro/compañeros, "el responsable principal es el tutor, recopila la documentación, reúne la información del profesorado, pregunta a cada profesor como funciona este niño, en que destaca o no y qué problemas tiene" (IES1). Esta responsabilidad es establecida por la Junta a través de un Protocolo en los siguientes términos: "El tutor debe detectar en clase alumnado con AACC a partir de un protocolo en primaria y secundaria con un cuestionario donde se detectan y evalúan; este 
protocolo pretende, de forma sistematizada, que todos los alumnos pasen un filtro". El profesorado de pedagogía terapéutica actúa partiendo de un "asesoramiento para buscar el material y orientar las actividades, lo aplica el profesor de área para normalizarlo" (CEIP2).

La falta de formación del profesorado para identificar y actuar con este alumnado es una evidencia, "los profesores están ejerciendo y tampoco tienen formación, se encuentran con leyes y normativas que no saben cómo aplicarlas ni las entienden" (ASO1). Los orientadores tampoco tienen formación al respecto, con lo que "están identificando trastornos cuando quizás no son derivadas de AACC, sino de problemas de salud mental, de gestión de las emociones o aburrimiento en clase, confundiéndose con TDH, falta de formación..." (ASO1). El psicólogo clínico también es responsable, debe detectar una AC, "el que haga esa evaluación se le pueden ir muchas cosas". La formación en sobredotación y talento es fundamental, "habría que indicar al profesor o al centro educativo que es lo que debe hacer, eso ya estaba en las instrucciones del 2015" (ASO1).

Debido a la falta de atención y diagnósticos poco acertados, los padres suelen recurrir a Asociaciones buscando un diagnóstico, "me llegan a través de la web preguntando si hay alguien que pueda evaluar a sus niños, casi diariamente y los derivamos al orientador del colegio" (ASO1). Otras veces son derivados a las Asociaciones por los propios Equipos de Orientación de zona para una repuesta respecto a los intereses del alumnado, "Esos niños vienen individualmente o vienen derivados por los Equipos de Orientación de los colegios o por los propios colegios porque nos conocen" (ASO2).

Las Asociaciones respetan el Informe elaborado por el Equipo de Orientación de Altas Capacidades: "si los niños ya vienen evaluados con por un EOE de Altas Capacidades se le admite en el Programa" (ASO2). Este alumnado debe ser evaluado por centros y profesionales que los atienden, "ya cada vez vienen más evaluados por los centros, se les ofrece la opción de beca y la posibilidad de hacer una actividad a nivel privado pagado por la beca del Ministerio" (ASO2).

Cod4. EMR: las estrategias de atención a la diversidad se basan en medidas curriculares, actividades de refuerzo y de ampliación, "es una adaptación del currículo y la programación" (IES1). Otra de las estrategias es la participación en "clases avanzadas", un desarrollo extra de la programación curricular, "se les entrega una documentación indicando lo que ha hecho y los temas tratados porque en esas clases avanzadas están fuera de currículum también" (IES2). Entre las medidas que utilizan los centros, algunas se desarrollan "a demanda" del propio alumnado, "trabajando sin ningún Programa específico, sin programación dirigida, va en las inquietudes que tiene, siguiendo los centros de interés del alumno" (CEIP1). Se amplían de forma libre sin encorsetamientos curriculares "pudiendo desarrollar sus capacidades de manera libre, (...), buscando material de forma autónoma cuando termina lo de clase" (CEIP2). En algunos centros existen programas como Profundiza, club de programación y estímulo del talento matemático.

En las Asociaciones, los programas y proyectos que desarrollan son muy variados, destacan el Proyecto Sandbox (caja de arena), Firstlego, campamentos emocionales, laboratorios, Talleres para padres, charlas de sensibilización, Programa PEPs (Estimulación para Principiantes, de 3 a 5 años), Programa Ingenia y de Robótica, Programa de enriquecimiento Cognitivo, Talleres de arqueología, matemáticas, Programa de radio y música, Feria de la Ciencia, "se diseñan unas actividades para trabajar la lógica, razonamiento numérico, verbal, espacial, bajo una temática, pero ellos trabajan con nuestro objetivo" (ASO2). Las Asociaciones elaboran Programas Avanzados en función de las demandas, "vamos elaborando los Programas en función de lo que los niños demandan como la temática, la metodología ya la tenemos establecida. Los temas se repiten, arqueología todos los años, pero siempre se tocan cosas distintas" (ASO2). 
Cod5. PFA: las familias constituyen un pilar importante en la atención a las AACC. Los centros trabajan con ellas en tutoría y Departamento de Orientación con entrevistas. Los padres no desean que su hijo sea etiquetado con AACC, en cursos inferiores opinan que es demasiado precoz esta detección, "un niño de primero de primaria donde inicia el proceso todavía es pronto"; "es demasiado pronto y prefieren que el niño era feliz y diagnosticar a partir de tercero" (CEIP1). En otros casos manifiestan inquietud, creen que el talento se pierde, "padres desesperados que ven cómo se pierde el talento o la capacidad de su hijo" (ASO2). "Hay familias que responden muy bien, otras que no quieren que su hijo sobresalga y se oponen o no participan, otras incluso creen que son más de lo que son" (CEIP2).

La familia actualmente tiene información, conoce el Plan de AACC de la Junta de Andalucía, presiona sobre el diagnóstico, "es la experiencia la que nos dice qué pasa, tanto en niños que destacan y luego se igualan y niños van por detrás y luego se igualan también" (CEIP1). Las Asociaciones ofrecen información y formación a las familias sobre este tema, "la familia necesita conocer algo más, llegan un poco asustados" (ASO1), buscan contacto con otros padres, las Asociaciones plantean Talleres para Padres, charlas de sensibilización y Escuela de Padres, "han venido para saber qué hacer, que nadie es raro, que todos son niños" (ASO1).

Cod6. OBB: entre los obstáculos podemos destacar que la atención al alumnado con AACC depende de varias cuestiones, la ratio y el cambio constante de plantilla de profesorado: "es mucho trabajo, muy complicado, cuesta sobre todo cuando la plantilla cambia. Depende mucho del profesor, de la sensibilidad. Estamos con una ratio muy alta..." (IES1). Existe alta demanda de evaluación de alumnado con AACC, con lo que los profesionales se encuentran colapsados para elaborar los Informes de Diagnóstico, "los padres tienen mucha información y vienen todos con el Plan de Altas Capacidades de la Junta, la presión la tenemos todos los orientadores, hay muchísima demanda de evaluación" (CEIP1).

\section{Conclusiones y Discusión}

Presentamos las conclusiones y su discusión, teniendo como referencia el objetivo principal del estudio y los objetivos secundarios. Varios son los aspectos que debemos comentar.

La necesidad de unificar criterios para conceptualizar las AACC como un objetivo prioritario a medio plazo. Este problema revierte en la conciliación de criterios. Nos referimos a coincidencias entre este alumnado, no a una relación de características, categorización y etiquetaje. Es muy importante considerar las diferencias individuales en las AACC ya que además del $\mathrm{Cl}$ hay que considerar otros tipos de talento y cualidades. El Modelo Tripartito de Pfeiffer (2015) es adecuado para abordar el diagnóstico de las AACC desde tres enfoques para su diagnóstico, es decir, inteligencia en las habilidades, logros sobresalientes en los resultados y potencial para rendir de modo excelente en las tareas.

La exigencia de abordar la variable de género, ya que también hay que considerar diferencias individuales en este sentido. Los profesionales ponen de manifiesto que las niñas con AACC pueden pasar desapercibidas porque tienden a adaptarse a las situaciones de clase poco motivadoras y a buscar "soluciones" al aburrimiento cuando no se atrae su atención. A diferencia de los niños que pueden tener comportamientos disruptivos cuando se aburren, con lo que algunos tienen más visibilidad en clase que las niñas. Parece que las niñas tienen recursos propios para estar activas, aunque podrían estar ajenas a la clase. 
La detección temprana de las AACC como tema prioritario, existiendo diversidad de opiniones en torno a la edad para realizar valoraciones tempranas debido a que el estudiante está en proceso madurativo. Higueras-Rodríguez (2017) afirma que el alumnado con AACC no solo no constituye un grupo homogéneo, sino que puede no mostrar todos sus rasgos definitorios o hacerlo de forma discontinua, dando lugar a su detección y evaluación insuficiente y tardía, y, hasta que ésta se produce, tanto familias como educadores manifiestan "angustia e incapacidad". Algunos centros desarrollan proyectos que sirven para detectar estudiantes con AACC y motivarlos para participar en programas concretos.

En relación con las familias, la opción por la atención educativa en centros ordinarios, complementada por Asociaciones. En estas se desarrollan programas específicos basados en intereses de los estudiantes y elaborados a demanda. Estos programas son variados (programas de enriquecimiento, talleres específicos, nuevas tecnologías, laboratorios específicos, campamentos emocionales, etc.). Las Asociaciones plantean una oferta formativa muy amplia para familias, centros educativos y profesorado, dada la escasa formación que reciben en los centros educativos, y cubriendo un "hueco" que no ofrece la Administración Educativa. Entre los retos principales de las Asociaciones se encuentra tener visibilidad a nivel escolar y social, ejercer una influencia mayor en las instituciones educativas y lograr la retroalimentación con los centros.

La responsabilidad del tutor/a de estos estudiantes en el centro, así como del profesorado y orientadores, los cuales manifiestan su soledad ante esta problemática respecto a su falta de formación e información. Agudo (2017) cuestiona y realiza propuestas sobre qué hacer cuando se tiene un estudiante con AACC en el aula. Las adaptaciones curriculares no parecen ser la respuesta educativa adecuada para este alumnado, supone realizar un trabajo extra que no les interesa. La atención escolar debe estar enfocada hacia trabajos útiles y compartidos con los demás en el aula, promoviendo la inclusión educativa, el sentido de pertenencia, participación y aceptación en el grupo.

Las dificultades del profesorado para una atención educativa individualizada y adecuada dentro del aula, las cuales son evidentes en los comentarios: "al profesorado dentro de clase le cuesta mucho trabajar con AACC cuando necesita refuerzo educativo" (IES-2). Entre los factores que condicionan la respuesta educativa figuran la falta de tiempo y la ratio. Fernández-Díez et al. (2017), plantean la necesidad de "explorar" al alumnado para atender su curiosidad, motivación o capacidad. La idea es paliar el comportamiento disruptivo de estos estudiantes: "hay niños que se portan mal porque se aburren" (IES-2). Las asignaturas de libre configuración constituyen un recurso valioso para este alumnado.

En relación con las Asociaciones especializadas en AACC, la socialización de los estudiantes que promueven, dando libertad de expresión y relacionándose con otros en igualdad. El problema de la "socialización" parece un mito asociado a las AACC que algunos autores han desmontado (Hernández y Gutiérrez, 2014). Los proyectos de estas Asociaciones tienen la finalidad de mejorar la convivencia, contribuyendo a promover las habilidades sociales, por lo que algunos Programas educativos que se organizan para trabajar con estos estudiantes están abiertos a otros estudiantes aventajados. El profesorado afirma que "algunos (estudiantes con AACC) han necesitado adelantar un curso y con esa adaptación ha sido suficiente, otros han necesitado un refuerzo en su creatividad" (IES-2). 


\section{Implicaciones para la orientación educativa}

Las Asociaciones actualmente colaboran con profesorado de centros privados y concertados en la elaboración de Programas de enriquecimiento, ofrecen a las familias información y atención individualizada en base a necesidades concretas, realizando tareas de sensibilización a nivel educativo, sanitario y universitario. El voluntariado con formación supone un recurso valioso para impartir talleres y cursos especializados. Algunas Asociaciones plantean como reto la atención específica a los/as adolescentes por tratarse de una etapa que puede ser conflictiva. La investigación de Cejudo et al. (2017), es un referente que asocia las inteligencias múltiples con inteligencia cognitiva y emocional en adolescentes.

Uno de los retos pendientes es la coordinación entre centros de educación primaria y secundaria mediante Programas de Tránsito, suponiendo un paso adelante que beneficia al profesorado, estudiantes con AC y familias por la continuidad de la atención educativa. Estos estudiantes pueden ejercer de "compañero tutor" y ayuda entre iguales, constituyendo un buen recurso para el Programa de Tránsito. Se realizan dinámicas de grupo que promueven las relaciones interpersonales y la socialización.

Hay mucho trabajo por realizar, organizar y mejorar en relación con las AACC, sobre todo la información que se ofrece a los sectores de la comunidad educativa, la necesidad de "desmontar" falsos mitos y creencias sobre las AACC (como ejemplo ser un estudiante con AACC no significa tener éxito a nivel escolar). En este sentido, la intervención orientadora específica a estos estudiantes es una tarea fundamental y relevante por lo que debería priorizarse en los centros educativos, por las implicaciones que tiene en la atención educativa adecuada y ajustada a las necesidades reales de estudiantes con AACC, considerando la singularidad y diversidad existente entre ellos. Esta orientación debería estar basada en criterios equitativos e inclusivos ya que podría ser la base del éxito de estos estudiantes. Asimismo, la formación de profesionales es uno de los retos más demandados y está contribuyendo a visibilizar las AACC, minimizando los mitos y las falsas creencias sobre este tema.

\section{Referencias bibliográficas}

Ainscow, M., Dyson, A., Goldrick, S. y West, M. (2013). Promoviendo la equidad en educación. Revista de Investigación en Educación, 11(3), 44-56.

Agudo, N. (2017). Un estudiante con altas capacidades en mi aula, ¿Ahora qué?, Revista Nacional e Internacional de Educación Inclusiva, 10(1), 265-277.

Albes, C., Aretxaga, L., Etxebarria, I., Galende, I., Santamaría, A., Uriarte, B. y Vigo, P. (2013). Orientaciones educativas. Alumnado con altas capacidades intelectuales. Departamento de Educación, Política Lingüística y Cultura. Gobierno Vasco.

Azorín, C. (2018). Abriendo fronteras para la inclusión: la Ecología de la equidad. Revista nacional e internacional de educación inclusiva, 11(1), 213-228. https://revistaeducacioninclusiva.es/index.php/REl/article/view/335

Bardín, L. (1996). Análisis de contenido. Akal (2ª ed). 
Cejudo, J., Losada, L. y Pérez-González, J. C. (2017). Inteligencias múltiples y su relación con inteligencia cognitiva y emocional en adolescentes, Universitas Psychologica, 16(3), 1-13. http://doi.org./10.11144/Javeriana.upsy16-3.imri

Clares, J. (2016). Metodología de Investigación Educativa. Proyectos de investigación. Un abordaje global desde la diversidad sus necesidades e intervención. AIECE.

Fernández-Batanero, J.M. (2020). Tareas de orientación y aprendizajes transversales. Innovación Educativa, 30, 07-20. https://doi.org/10.15304/ie.30.7062

Ferrer, A. (2019). Identificando buenas políticas y prácticas para una educación inclusiva. Participación Educativa, 6(9), 165-174.

https://sede.educacion.gob.es/publiventa/identificando-buenas-politicas-y-practicas-parauna-educacion-inclusiva/ensenanza-politica-educativa/23961

Fernández-Díez, J., Cencerrado-de-Aller, R., Rodríguez-Fernández, J., Hierro-Berceo, A., Sánchez-Fernández, E. y Pérez-Sáenz, J. (2017). Explora. Atender a los alumnos con más curiosidad, motivación o capacidad sin excluir a nadie. Psicología Educativa, 23, 95-103. http://dx.doi.org/10.1016/i.pse.2017.05.0044

Gagné, F. (1991). Toward a Differentiated Model of Giftedness and Talent. En N. Collangelo y G.A. Davis (Eds.) Handbook of Gifted Education (pp. 65-80). Allyn and Bacon.

Garcerán, M.C. (2019). Altas capacidades, educación y orientación familiar. Almoraima. Revista de Estudios Campo-Gibraltareños, 50, 159-171.

http://institutoecg.es/wp-content/uploads/2019/05/Altas-capacidades.pdf

García, R. (2016). Desempeño de los más capaces para la matemática en la prueba de rendimiento BECOMA: Correlación de los resultados con el test psicométrico BADYG-E3. Revista Española de Orientación y Psicopedagogía, 27(2), 45-61. https://doi.org/10.5944/reop.vol.27.num.2.2016.17113

Gardner, R.C. (1985). Social Psychology and Second Language Learning: The Role of Attitude and Motivation. Edward Arnold.

Gonzalez-Gil, F., Martín-Pastor, E. y Poy, R. (2019). Educación inclusiva: barreras y facilitadores para su desarrollo. Análisis de la percepción del profesorado. Profesorado. Revista de

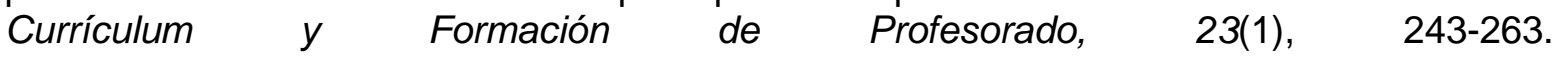
http://doi.org./10.30827/profesorado.v23i1.9153

Gronostaj, A., Werner, E., Bochow, E. y Vock, M. (2016). How to learn things at school you don't already know: experiences of gifted grade-skippers in Germany. Gifted Child Quarterly, 60(1), 31-46. https://doi.org/10.1177/0016986215609999

Guirado, A. (2015). ¿Qué sabemos sobre las altas capacidades? Graó.

Hernández, D. y Gutiérrez, M. (2014). El estudio de la alta capacidad intelectual en España: Análisis de la situación actual. Revista de Educación, 364, 251-272. https://sede.educacion.gob.es/publiventa/el-estudio-de-la-alta-capacidad-intelectual-enespana-analisis-de-la-situacion-actual/investigacion-educativa/16368

Hernández-Sampieri, R., Fernández, C., y Baptista, P. (2014). Metodología de la Investigación. McGraw-Hill.

Higueras-Rodríguez, L. (2017). Intervención educativa en el alumnado con altas capacidades. Ensayos Pedagógicos, 12(1), 69-81. http://dx.doi.org./10.15359/rep.12-14 
Morales, R., Navarro, M. y Rondón, B. (2017). Perspectivas de la orientación educativa: retos e intervención en el área de las altas capacidades y desarrollo de talentos estudiantiles. Revista Ciencias de la Educación, 27(50), 58-72.

http://servicio.bc.uc.edu.ve/educacion/revista/50/art04.pdf

Instrucciones de 22 de junio de 2015, de la Dirección General de Participación y Equidad de la Junta de Andalucía.

Kim, E. (2014). La detección y el diagnóstico de las altas capacidades. Instituto Internacional de Altas Capacidades. http://altascapacidadescse.org/LaDeteccionyelDiagnostico.pdf

Kim, M. (2016). A meta-analysis of the effects of enrichment programs on gifted students. Gifted Child Quarterly, 60(2), 102-116. https://doi.org/10.1177/0016986216630607

Kroesbergen, E., van Hooijdonk, M., Van Viersen, S., Middel-Lalleman, M. y Reijnders, J. (2016). The psychological well-being of early identified gifted children. Gifted Child Quarterly. 60(1),16-30. https://doi.org/10.1177/0016986215609113

Ley Orgánica 1/1990, de 3 de octubre, de Ordenación General del Sistema Educativo, LOGSE. (BOE, 238, de 4 de octubre de 1990).

Ley Orgánica 10/2002, de 23 de diciembre, de Calidad de la Educación, LOCE (BOE, 24/12/2002).

Ley Orgánica 2/2006, de 3 de mayo, de Educación, LOE. (BOE, 04/05/06).

Martínez, M. y Guirado, A. (Coords.) (2014). Altas capacidades intelectuales. Graó.

McClarty, K. (2015). Life in the fast lane: effects of early grade acceleration on high school and college outcomes. Gifted Child Quarterly, 59(1), 3-13. https://doi.org/10.1177/0016986214559595

MECD (2016). Las cifras de la educación en España. Curso 2013-2014 (Edición 2016), Subdirección General de Estadística y Estudios del Ministerio de Educación, Cultura y Deporte. Gobierno de España.

https://www.educacion.gob.es/educabase/menu.do?type=pcaxis\&path=/Educacion/Alumna do/Apoyo/Curso13-14/AltasCapacidades\&file=pcaxis\&l=s0

Mönks, F.J. (1992). Development of Gifted Children: The Issue of Identification and Programing. En W.A.M. Peters, Talent for the Future, Van Gorcum.

Mudrak, J. y Zabrodska, K. (2015). Chidhood giftedness, adolescent agency: a systemic multiplecase study. Gifted Child Quarterly, 59(1), 55-70.

https://doi.org/10.1177/0016986214559602

Núñez del Río, M. C., Biencinto, Ch., Carpintero, E. y García, M. (2014). Enfoques de atención a la diversidad, estrategias de aprendizaje y motivación en Educación Secundaria. Perfiles Educativos, Vol. XXXVI (145), 65-80. https://www.redalyc.org/articulo.oa?id=13231362005

Orozco, I y Moriña, A. (2019). Prácticas docentes para una Pedagogía Inclusiva en Educación Primaria: Escuchando las voces del profesorado. Aula Abierta, 4(3), 331-338. https://doi.org/10.17811/rifie.48.3.2019.331-338

Palomares, A. y García, R. (2016). Innovación y creatividad para favorecer la intervención educativa del alumnado con altas capacidades. Revista de Educación Inclusiva, 9(1), 90100. https://revistaeducacioninclusiva.es/index.php/REl/article/view/73/70 
Pfeiffer, S. (2015). El Modelo Tripartito sobre la alta capacidad y las mejores prácticas en la evaluación de los más capaces. Revista de Educación, 368, 66-95. https://doi.org/10.4438/1988-592X-RE-2015-368-293

Plan de Actuación para la atención educativa al alumnado con necesidades específicas de apoyo educativo por presentar altas capacidades intelectuales en Andalucía (2011), Consejería de Educación Junta de Andalucía. Dirección General de Participación e Innovación Educativa.

Plucker, J. y Callahan, C. (2014). Research on giftedness and gifted education: status of the field and considerations for the future. Exceptional Children, 80(4), 390-406. https://doi.org/10.1177/0014402914527244

Ramos, J. A. (2014). La paradoja del sistema educativo. Su naturaleza incluyente/excluyente. Perfiles Educativos, Vol. XXXVI (146), 154-173.

Rasmussen, A. y Rasmussen, P. (2015). Conceptions of Student Talent in the Context of Talent Development. International Journal of Qualitative Studies in Education, 28(4) 476-495. https://doi.org/10.1080/09518398.2014.916013

Sáenz de Jubera, M. M. y Chocarro, E. (2019). La atención a la diversidad desde la perspectiva del profesorado. Revista Mejicana de Investigación Educativa, 24(82), 89-809.

Salmela, M. y Määttä, K. (2015). Even the best have difficulties: A study of Finnish Straight-A Graduate's Resource-oriented Solutions. Gifted Child Quarterly, 59 (2), 124-135. https://doi.org/10.1177/0016986214568720

Sánchez, A. (2013). Altas capacidades intelectuales: sobredotación y talentos. Formación Alcalá.

Sánchez A. S. y Baena, M. J. (2017). Identificación del alumno con altas capacidades intelectuales: ¿responsabilidad del maestro o del departamento de orientación educativa y psicopedagógica? Aula de Encuentro, 19(1), 69-91. http://hdl.handle.net/11162/157787, https://redined.mecd.gob.es/xmlui/handle/11162/157787

Sastre-Riba, S. (2014). Intervención psicoeducativa en la alta capacidad: funcionamiento intelectual y enriquecimiento extracurricular. Revista de Neurología, 58(1), 89-98.

Seaton, M., Marsh, H., Parker, P., Craven, R. y Yeung, A. (2015). The reciprocal effects model revisited: extending its reach to gifted students attending academically selective schools. Gifted Child Quarterly, 59(3), 143-156. https://doi.org/10.1177/0016986215583870

Sternberg, R.J. (1985). Beyond IQ: A Triarchic Theory of intelligence. Cambridge University Press.

Subotnik, R., Olszewski-Kubilius, P. y Worrell, F. (2012). Rethinking Giftedness and Gifted Education a Proposed Direction Forward Based on Psychological Science. Psychological Science in the Public Interest, 12(1), 3-54. https://doi.org/10.1177/1529100611418056

Tannenbaum, A.J. (1986). Giftedness: a Psychosocial Approach. En R.J Sternberg y J.E. Davidson (Eds.). Conceptions of Giftedness (pp. 21-52). Cambridge University Press.

Tannenbaum, A.J. (1997). The meaning and making of giftedness. En N. Colangelo y G. Davis (Eds.) Handbook of Gifted Education. 2ª ed (pp. 27-43). Allyn \& Bacon.

Terman, L. (1917). The intelligence quotient of Francis Galton in childhood. American Journal of Psychology, 28(2), 209-215.

Torrego, J. C. (coord.) (2012). Alumnos con altas capacidades y aprendizaje cooperativo. Pryconsa, Fundación SM. 
Touron, J. y Touron, M. (2011). The Center for Talented Youth Identification Model: A Review of the Literature. Talent Development and Excellence, 3, 187-202. https://dadun.unav.edu/handle/10171/19605, https://hdl.handle.net/10171/19605

Vallejo, P. y Morata, M. (2015). Intervención psicoeducativa en un caso de altas capacidades. Revista de Psicología Clínica con Niños y Adolescentes, 2(1), 69-74. https://www.redalyc.org/articulo.oa?id=477147185009

Velasco, M. F. y Quiroga-Garza, A. (2018). Factores sistémicos asociados a la experiencia escolar de adolescentes con alto potencial intelectual. Revista Mejicana de Investigación Educativa, 23(79), 1051-1074.

https://pure.udem.edu.mx/en/publications/factores-sist\%C3\%A9micos-asociados-a-laexperiencia-escolar-de-adolesc

Fecha de entrada: 21 abril 2020

Fecha de revisión: 16 octubre 2020

Fecha de aceptación: 13 noviembre 2020 